\title{
Efficacy and Safety of Adding Sitagliptin in Type 2 Diabetes Patients on Insulin: Age-Stratified Comparison at One Year in the ASSIST-K Study
}

\author{
Masahiko Takai ${ }^{\mathrm{a}}$, Masashi Ishikawa ${ }^{\mathrm{a}}$, Hajime Maeda ${ }^{\mathrm{a}}$, Akira Kanamori ${ }^{\mathrm{a}}$, Akira Kubota ${ }^{\mathrm{a}}$, Hikaru Amemiya ${ }^{\mathrm{a}}$, \\ Takashi Iizuka $^{a}$, Kotaro Iemitsu ${ }^{a}$, Tomoyuki Iwasaki ${ }^{a}$, Goro Uehara ${ }^{a}$, Shinichi Umezawa ${ }^{a}$, Mitsuo Obana ${ }^{a}$, \\ Hideaki Kaneshige ${ }^{a}$, Mizuki Kaneshiro ${ }^{a}$, Takehiro Kawata ${ }^{a}$, Nobuo Sasai ${ }^{a}$, Tatsuya Saito ${ }^{\text {a }}$ Tetsuo Takuma ${ }^{a}$, \\ Hiroshi Takeda ${ }^{a}$, Keiji Tanaka ${ }^{a}$, Shigeru Nakajima ${ }^{a}$, Kazuhiko Hoshino ${ }^{a}$, Shin Honda ${ }^{a}$, Hideo Machimura ${ }^{a}$, \\ Kiyokazu Matoba a, Fuyuki Minagawa ${ }^{a}$, Nobuaki Minamia, Yukiko Miyairia, Atsuko Mokubo ${ }^{a}$, Tetsuya \\ Motomiya $^{a}$, Manabu Waseda ${ }^{\text {a }}$, Masaaki Miyakawa ${ }^{a}$, Yasuo Terauchi ${ }^{b}$, \\ Yasushi Tanakac , Ikuro Matsuba a, d, e
}

\begin{abstract}
Background: Sitagliptin, the first dipeptidyl peptidase- 4 inhibitor, has demonstrated efficacy and safety as monotherapy and as add-on therapy to oral antidiabetic agents or insulin. However, there have been few reports about sitagliptin in elderly patients. The ASSIST-K observational study was performed in patients with type 2 diabetes mellitus (T2DM) receiving sitagliptin as add-on therapy to insulin. Changes of hemoglobin A1c (HbA1c), body weight, and the estimated glomerular filtration rate (eGFR), as well as adverse events, were investigated over 12 months in age-stratified groups.
\end{abstract}

Methods: Among outpatients with T2DM treated at member institutions of Kanagawa Physicians Association, those starting sitagliptin as add-on therapy to insulin were followed for 12 months. HbA1c (National Glycohemoglobin Standardization Program), body weight, and eGFR were the efficacy endpoints, while adverse events were investigated to assess safety. Patients were stratified into three age groups ( $\leq$ 64 years, 65 - 74 years, and $\geq 75$ years) for comparison of the endpoints.

Results: Among 937 patients on insulin before starting sitagliptin, 821 patients were analyzed after excluding those without $\mathrm{HbA} 1 \mathrm{c}$ data at baseline and 12 months. The two groups of elderly patients (65- 74 years and $\geq 75$ years) had more complications and their HbA1c was

Manuscript submitted November 2, 2018, accepted February 18, 2019

aStudy Group of the Diabetes Committee, Kanagawa Physicians Association, Kanagawa, Japan

${ }^{b}$ Department of Endocrinology and Metabolism, Yokohama City University, Kanagawa, Japan

'Department of Internal Medicine, Division of Metabolism and Endocrinology, St. Marianna University School of Medicine, Kanagawa, Japan

dMatsuba Medical Clinic, Kanagawa, Japan

${ }^{e}$ Corresponding Author: Ikuro Matsuba, Matsuba Medical Clinic, 2-159 Tsukagoshi, Saiwai-ku, Kawasaki-shi, Kanagawa 212-0024, Japan.

Email: ikuro@matsuba-web.com

doi: https://doi.org/10.14740/jocmr3677 lower at initiation of sitagliptin therapy. The dose of sitagliptin, daily number of insulin injections, and number of concomitant oral antidiabetic agents were all lower in the elderly patients. HbA1c showed a significant decrease after initiation of sitagliptin in all age groups, and there were no significant intergroup differences in the change of $\mathrm{HbA1c}$ at 12 months. Body weight did not change significantly in any group. eGFR decreased significantly in all groups, with no significant intergroup differences at 12 months. Regarding adverse events, there were no significant intergroup differences in the incidence of severe hypoglycemia, gastrointestinal symptoms, or constipation.

Conclusions: Despite baseline differences in demographic factors and medications, sitagliptin showed good efficacy and safety in all age groups of patients receiving it as add-on therapy to insulin during routine management of T2DM. Adding sitagliptin to insulin achieves similar efficacy and safety outcomes at 12 months in both elderly and non-elderly T2DM patients.

Keywords: Type 2 diabetes mellitus; Dipeptidyl peptidase- 4 inhibitor; Sitagliptin; Hemoglobin A1c

\section{Introduction}

Dipeptidyl peptidase-4 (DPP-4) inhibitors are oral hypoglycemic agents (OHAs) that increase endogenous incretin levels and promote insulin secretion in a blood glucose-dependent manner by selectively inhibiting DPP-4, an enzyme that degrades incretins (glucagon-like peptide 1 and glucose-dependent insulinotropic polypeptide) [1]. In 2017, nine DPP-4 inhibitors were available in Japan, including two for once-weekly administration $[2,3]$. Meta-analyses have not found any significant differences in the hypoglycemic effect of different DPP-4 inhibitors $[4,5]$. These drugs have a favorable safety profile that is characterized by a low risk of hypoglycemia and weight gain [6]. Sitagliptin was the first DPP-4 inhibitor to become available clinically and it was launched in Japan in 2009 [7]. 
Its efficacy has been confirmed as monotherapy for drug-naive patients and as add-on therapy to other OHAs or insulin [8].

To assess the efficacy and safety of sitagliptin in the setting of routine management of diabetes by diabetologists, we investigated the 12-month course after initiation of sitagliptin therapy in outpatients with poorly controlled type 2 diabetes mellitus (T2DM). We have already reported the results obtained in patients not receiving concomitant insulin therapy (ASSET-K study) [9-14] and in patients receiving insulin (ASSIST-K study) [15, 16]. In addition, a related study (ATTEST-K study) was conducted in T2DM outpatients not receiving insulin who were managed by non-diabetologists, and factor analysis of the 12-month changes in hemoglobin A1c (HbA1c), body weight, and estimated glomerular filtration rate (eGFR) was performed using combined data from all three studies (ASSET-K, ASSIST-K, and ATTEST-K) [17].

Compared with younger patients, elderly T2DM patients have a higher risk of developing complications of diabetes (microangiopathy and macroangiopathy), osteoporosis, and dementia $[18,19]$; and their mortality rate was reported to be twice that of non-diabetic elderly persons [20]. Since the incidence of hypoglycemia is also higher in elderly T2DM patients, careful patient education and prudent drug selection are required to provide appropriate treatment [21, 22]. However, there have only been a few reports about the efficacy and safety of sitagliptin in elderly patients with T2DM.

We previously reviewed the ASSET-K study data for patients not receiving insulin and found that add-on sitagliptin therapy demonstrated comparable efficacy and safety in elderly T2DM patients ( $\geq 75$ years old) compared to non-elderly patients [14]. In the present study, we stratified patients from the ASSIST-K study into three age groups to investigate the efficacy of add-on sitagliptin therapy in elderly T2DM patients receiving insulin, based on the changes in $\mathrm{HbAlc}$, body weight, and eGFR over 12 months, as well reviewing adverse events to assess safety.

\section{Materials and Methods}

\section{Study design}

The ASSIST-K study was a 1-year multicenter observational study conducted at member institutions of Kanagawa Physicians Association that specialized in managing diabetes.

\section{Subjects}

Outpatients with T2DM over 20 years old attending member institutions of Kanagawa Physicians Association were eligible to be enrolled in this study if they had shown poor glycemic control on insulin therapy for at least 1 month before initiation of sitagliptin. The following patients were excluded: patients with a history of hypersensitivity to any of the ingredients of sitagliptin; patients who had experienced severe ketoacidosis, diabetic coma, or precoma within 6 months before starting sitagliptin; patients with severe infection; patients in the preop- erative or postoperative period; patients with severe trauma; patients using glinides; and other patients who were judged to be inappropriate for this study by the investigator.

\section{Endpoints}

Demographic factors were investigated, including the sex, age, height, duration of diabetes, family history, smoking, alcohol intake, and medical complications. Treatment with other antidiabetic agents was assessed before initiation of sitagliptin, at the start of add-on sitagliptin therapy, and after 12 months of sitagliptin treatment. The following efficacy endpoints were examined at each specified time point: HbA1c (National Glycohemoglobin Standardization Program), blood glucose (fasting/ postprandial), body weight, blood pressure (systolic/diastolic), liver function parameters (glutamate oxaloacetate transaminase, glutamate pyruvate transaminase, and gamma-glutamyl transpeptidase), renal function parameters (serum creatinine and eGFR), serum lipids (total cholesterol, low-density lipoprotein cholesterol, high-density lipoprotein cholesterol, and triglycerides), and serum amylase. As the safety endpoint, adverse events were investigated at each specified time point.

\section{Statistical analysis}

Among patients enrolled in the ASSIST-K study, those receiving sitagliptin as add-on therapy to insulin were analyzed if they had HbA1c data at both the start and after 12 months of sitagliptin treatment (i.e., the population in which the change in $\mathrm{HbA1c}$ over 12 months could be calculated).

Appropriate descriptive statistics were calculated for demographic factors, such as sex and diabetic complications, in all patients using insulin before starting sitagliptin and in the patients with data allowing the 12-month change in $\mathrm{HbA} 1 \mathrm{c}$ to be calculated. Fisher's exact test or the $t$-test was used for comparisons stratified by age ( $\leq 64$ years, $65-74$ years, and $\geq 75$ years) among the patients in whom the 12-month change in HbA1c could be calculated.

Fisher's exact test or the Wilcoxon test was conducted for age-stratified comparison of the daily number of insulin injections, use of other oral antidiabetic agents (sulfonylureas, biguanides, thiazolidinediones, alpha-glucosidase inhibitors, and glinides), and number of antidiabetic agents used before initiation of add-on sitagliptin therapy, at the start of sitagliptin therapy, and after 12 months of sitagliptin treatment. Descriptive statistics were calculated for the daily dose of sitagliptin at the start and after 12 months of add-on therapy, and the Wilcoxon test was employed for comparison between age groups.

Graphs were prepared that displayed $\mathrm{HbA1c}$, body weight, and eGFR at the start of add-on sitagliptin therapy and after 3, 6 , and 12 months of treatment, as well as graphs showing the changes at each time point, and the one-sample and two-sample $t$-tests were carried out for intra-group and inter-group comparisons. Fisher's exact test was used to compare the occurrence of adverse events (severe hypoglycemia, gastrointestinal symptoms, constipation, and other events) between age groups.

$P$ values less than 0.05 were considered to be statistically 
Table 1. Baseline Demographic Factors: All Patients on Insulin Receiving Add-on Sitagliptin Versus Patients With Sufficient HbA1c Data

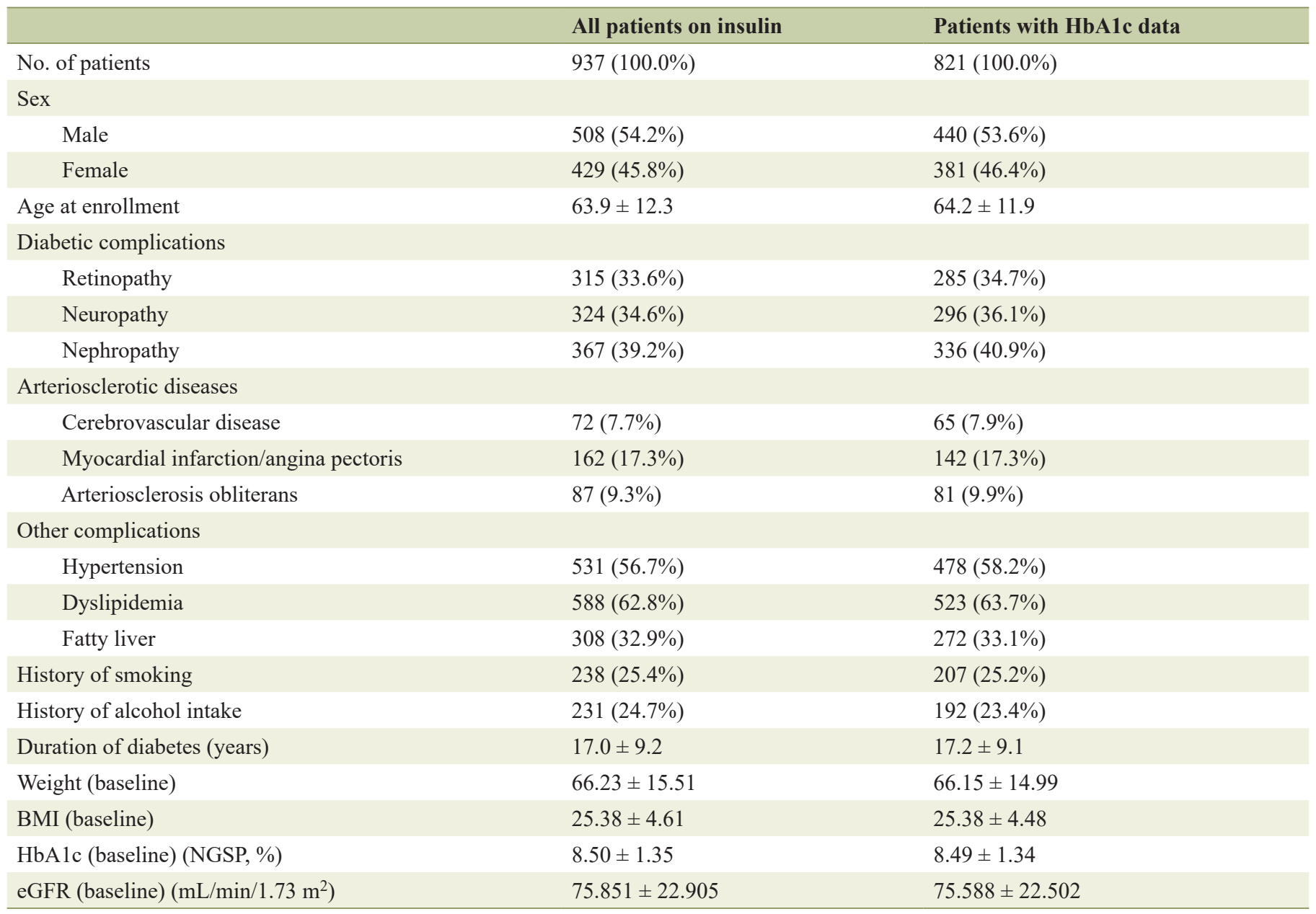

Men: eGFR $=194 \times$ serum creatinine ${ }^{-1.094} \times$ age at enrollment ${ }^{-0.287}$; Women: eGFR $=194 \times$ serum creatinine $^{-1.094} \times$ age at enrollment $0.287 \times 0.739$. Data are shown as the mean \pm standard deviation unless otherwise noted. BMI: body mass index; eGFR; estimated glomerular filtration rate; HbA1c: hemoglobin A1c; NGSP: National Glycohemoglobin Standardization Program.

significant.

\section{Results}

\section{Subjects}

Of the 1,168 patients enrolled in the ASSIST-K study, 937 patients were using insulin before initiation of add-on sitagliptin therapy. Among them, HbA1c was measured at the start of sitagliptin therapy and after 12 months in 821 patients (389 aged $\leq 64$ years, 267 aged $65-74$ years, and 165 aged $\geq 75$ years), who were subjected to detailed analysis.

\section{Demographic factors}

Table 1 shows the demographic profile of the 937 patients us- ing insulin before initiation of sitagliptin therapy and the 821 patients in whom HbA1c was measured at the start and after 12 months of sitagliptin treatment. All of the items assessed displayed a comparable distribution in the two patient populations.

In the 821 patients with complete HbA1c data, demographic factors were compared among the three age groups $(\leq$ 64 years, $65-74$ years, and $\geq 75$ years). The proportion of men was lower in the elderly group aged 65 - 74 years $(47.2 \%)$ and the very elderly group aged $\geq 75$ years $(46.7 \%)$ compared with the non-elderly group aged $\leq 64$ years $(60.9 \%)$ (Table 2$)$. In addition, the frequency of diabetic complications (retinopathy, neuropathy, and nephropathy) was higher in the two elderly groups (65 - 74 years and $\geq 75$ years) compared with the non-elderly group. The prevalence of arteriosclerotic diseases (cerebrovascular disease, myocardial infarction/angina pectoris, and arteriosclerosis obliterans) and other complications (hypertension, hyperlipidemia, and fatty liver) was also higher in the two elderly groups, but the rates of drinking and smok- 
Table 2. Demographic Factors of Each Age Group

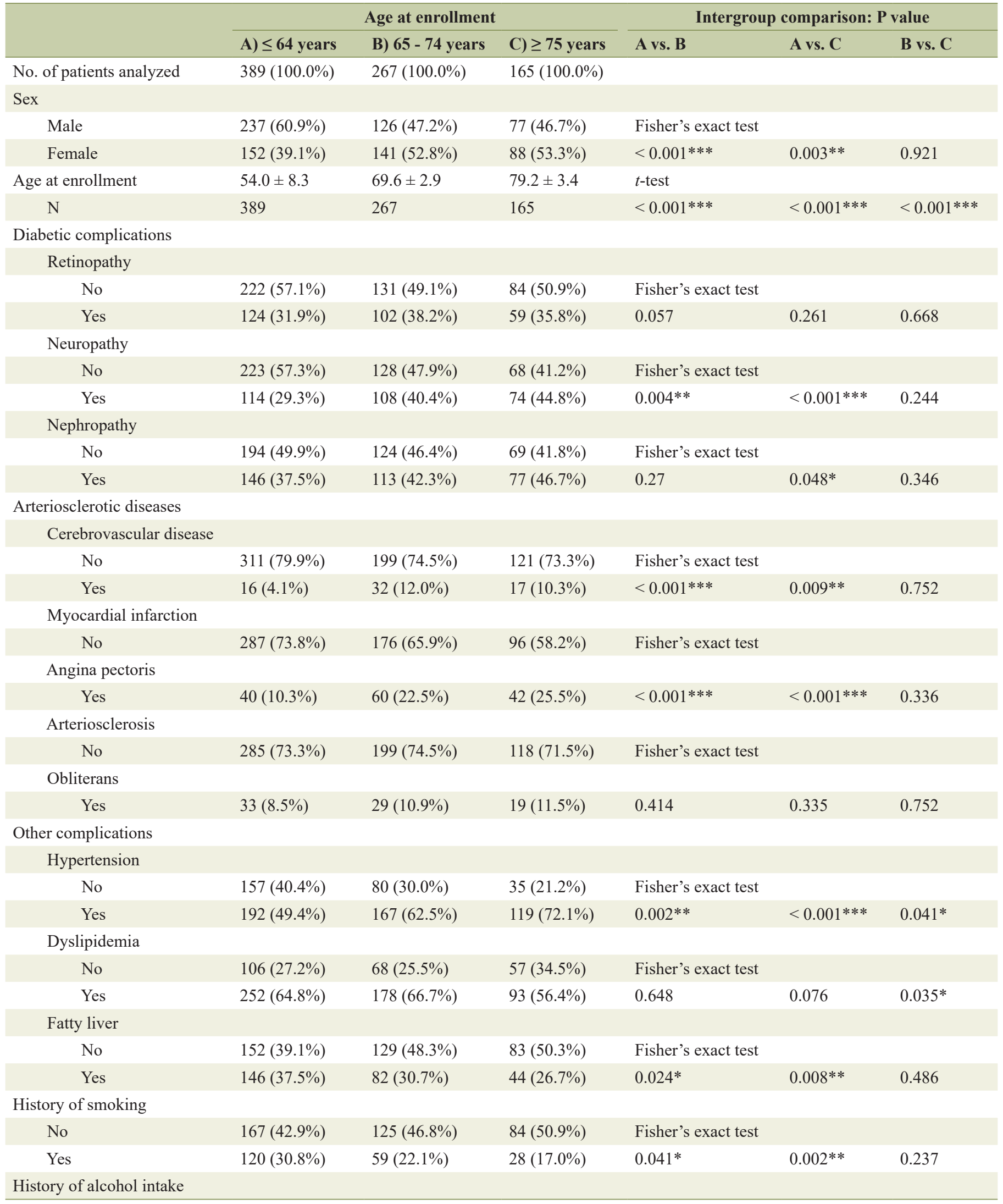


Table 2. Demographic Factors of Each Age Group - (continued)

\begin{tabular}{|c|c|c|c|c|c|c|}
\hline & \multicolumn{3}{|c|}{ Age at enrollment } & \multicolumn{3}{|c|}{ Intergroup comparison: $P$ value } \\
\hline & A) $\leq 64$ years & B) 65 - 74 years & C) $\geq 75$ years & A vs. B & A vs. C & B vs. C \\
\hline No & $165(42.4 \%)$ & $113(42.3 \%)$ & $90(54.5 \%)$ & Fisher's exact test & & \\
\hline Duration of diabetes (years) & $13.6 \pm 7.2$ & $19.8 \pm 8.6$ & $21.3 \pm 10.5$ & $t$-test & & \\
\hline $\mathrm{N}$ & 331 & 227 & 141 & $<0.001 * * *$ & $<0.001 * * *$ & 0.123 \\
\hline BMI (baseline) & $26.58 \pm 4.81$ & $24.55 \pm 3.74$ & $23.89 \pm 4.03$ & $t$-test & & \\
\hline $\mathrm{N}$ & 376 & 256 & 159 & $<0.001 * * *$ & $<0.001 * * *$ & 0.089 \\
\hline HbA1c (baseline) (NGSP, \%) & $8.80 \pm 1.50$ & $8.24 \pm 1.09$ & $8.19 \pm 1.13$ & $t$-test & & \\
\hline $\mathrm{N}$ & 389 & 267 & 165 & $<0.001 * * *$ & $<0.001 * * *$ & 0.644 \\
\hline
\end{tabular}

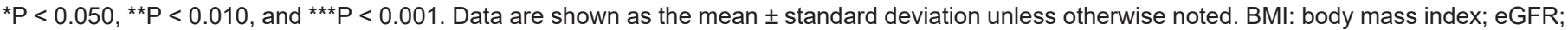
estimated glomerular filtration rate; HbA1c: hemoglobin A1c; NGSP: National Glycohemoglobin Standardization Program.

ing were lower. The mean duration of diabetes was 13.6 years in the non-elderly group, 19.8 years in the elderly group, and 21.3 years in the very elderly group, showing an increase in both elderly groups. In contrast, mean body weight was lower and mean body mass index was smaller in the elderly groups. Mean baseline HbA1c was $8.80 \%$ in the non-elderly group, $8.24 \%$ in the elderly group, and $8.19 \%$ in the very elderly group, being lower in the elderly groups. Mean baseline eGFR $\left(\mathrm{mL} / \mathrm{min} / 1.73 \mathrm{~m}^{2}\right)$ was 85.1 in the non-elderly group, 70.0 in the elderly group and 63.2 in the very elderly group, showing a decrease in elderly groups.

\section{Antidiabetic agents}

Antidiabetic agents used by the patients are summarized in Table 3 and are compared between the age groups. Before initiation of sitagliptin therapy (baseline), the mean daily frequency of insulin administration was 2.8 times in the nonelderly group, 2.7 times in the elderly group, and 2.4 times in the very elderly group, and it was significantly lower in the very elderly group. The mean number of OHAs (other than sitagliptin) was 1.1 in the non-elderly group, 1.0 in the elderly group, and 0.8 in the very elderly group, also being significantly lower in the very elderly group. Among OHAs, biguanides were used by $51.4 \%$ of the non-elderly group, $36.7 \%$ of the elderly group, and $23.6 \%$ the very elderly group, with the frequency of prescription decreasing in the two elderly groups.

At initiation of sitagliptin therapy (baseline), the mean dose of sitagliptin was $47.1 \mathrm{mg}$ in the non-elderly group, 45.1 $\mathrm{mg}$ in the elderly group, and $45.0 \mathrm{mg}$ in the very elderly group, being significantly higher in the non-elderly group. The daily frequency of insulin administration at baseline was similar to that before baseline. The mean number of concomitant OHAs was decreased at baseline in all age groups (1.0 in the nonelderly group, 0.8 in the elderly group, and 0.6 in the very elderly group). Concomitant use of the following OHAs decreased by at least 3\%: thiazolidinediones $(13.9 \%$ to $8.5 \%$ ) and alpha-glucosidase inhibitors $(26.2 \%$ to $19.0 \%)$ in the nonelderly group, thiazolidinediones $(9.0 \%$ to $4.9 \%)$ and alphaglucosidase inhibitors (26.6\% to $21.3 \%$ ) in the elderly group, and biguanides $(23.6 \%$ to $18.8 \%)$, alpha-glucosidase inhibitors ( $26.1 \%$ to $18.2 \%)$, and glinides $(3.0 \%$ to $0.0 \%)$ in the very elderly group.

After 12 months of sitagliptin treatment, the mean dose of sitagliptin was increased in all age groups $(52.8 \mathrm{mg}$ in the non-elderly group, $51.7 \mathrm{mg}$ in the elderly group, and 50.3 $\mathrm{mg}$ in the very elderly group). While the mean daily number of insulin injections was almost unchanged in each group, the mean number of concomitant OHAs showed a decrease from baseline ( 0.9 in the non-elderly group, 0.7 in the elderly group, and 0.6 in the very elderly group). Among concomitant OHAs, use of biguanides was decreased by at least 3\% versus baseline in all age groups (from 50.4\% to $44.2 \%$ in the non-elderly group, $35.2 \%$ to $27.7 \%$ in the elderly group, and $18.8 \%$ to $15.2 \%$ in the very elderly group), while use of sulfonylureas was reduced by $\geq 2 \%$ in the non-elderly and elderly groups (from 19.5 to 17.0 and $22.5 \%$ to $17.6 \%$, respectively), but increased in the very elderly group (from $18.8 \%$ to $21.8 \%$ ).

\section{Changes of HbA1c}

The HbAlc profile after initiation of sitagliptin treatment is displayed in Figure 1. After 12 months, mean HbAlc was reduced from $8.80 \%$ to $8.18 \%$ in the non-elderly group, from 
Table 3. Antidiabetic Agents

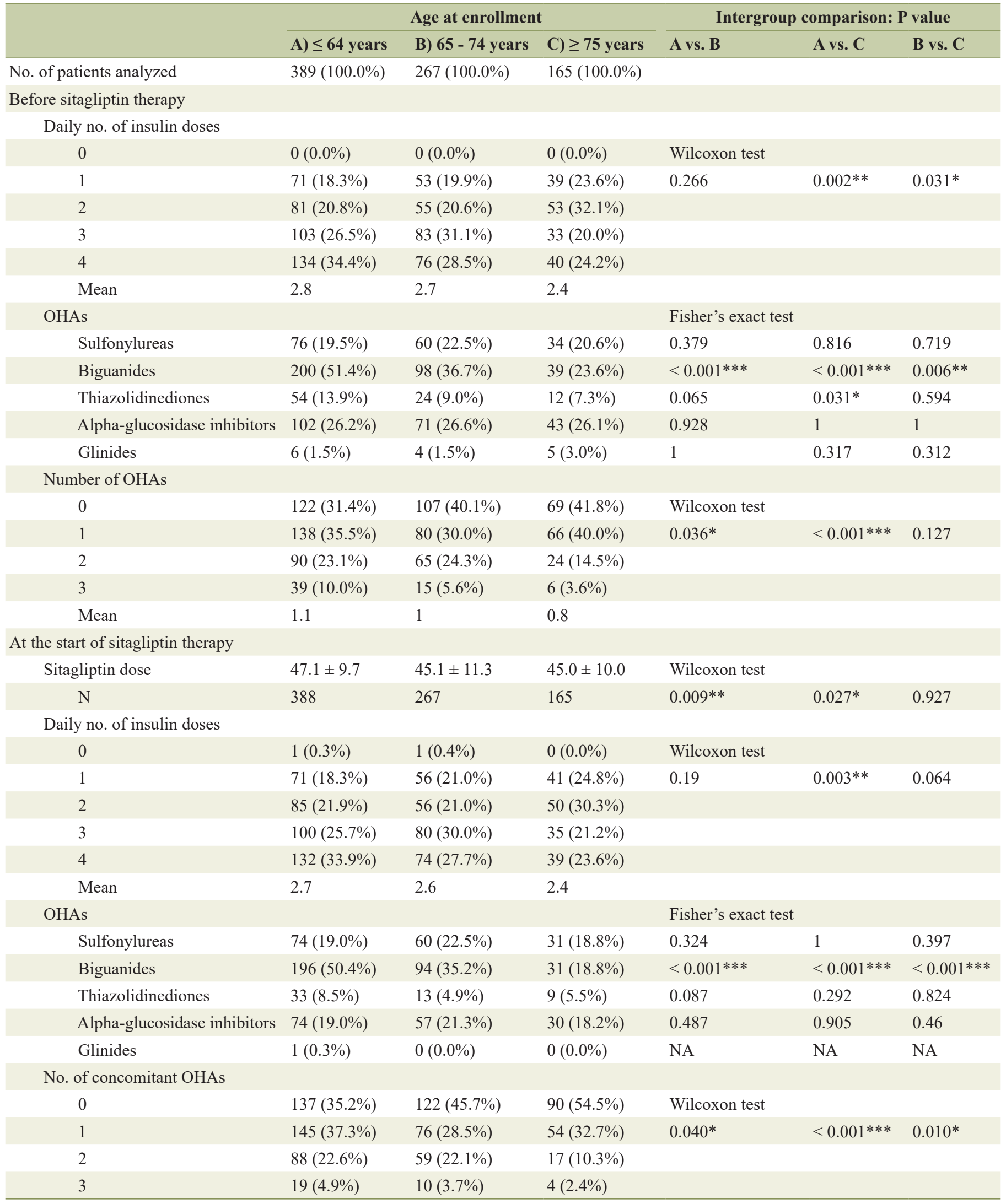


Table 3. Antidiabetic Agents - (continued)

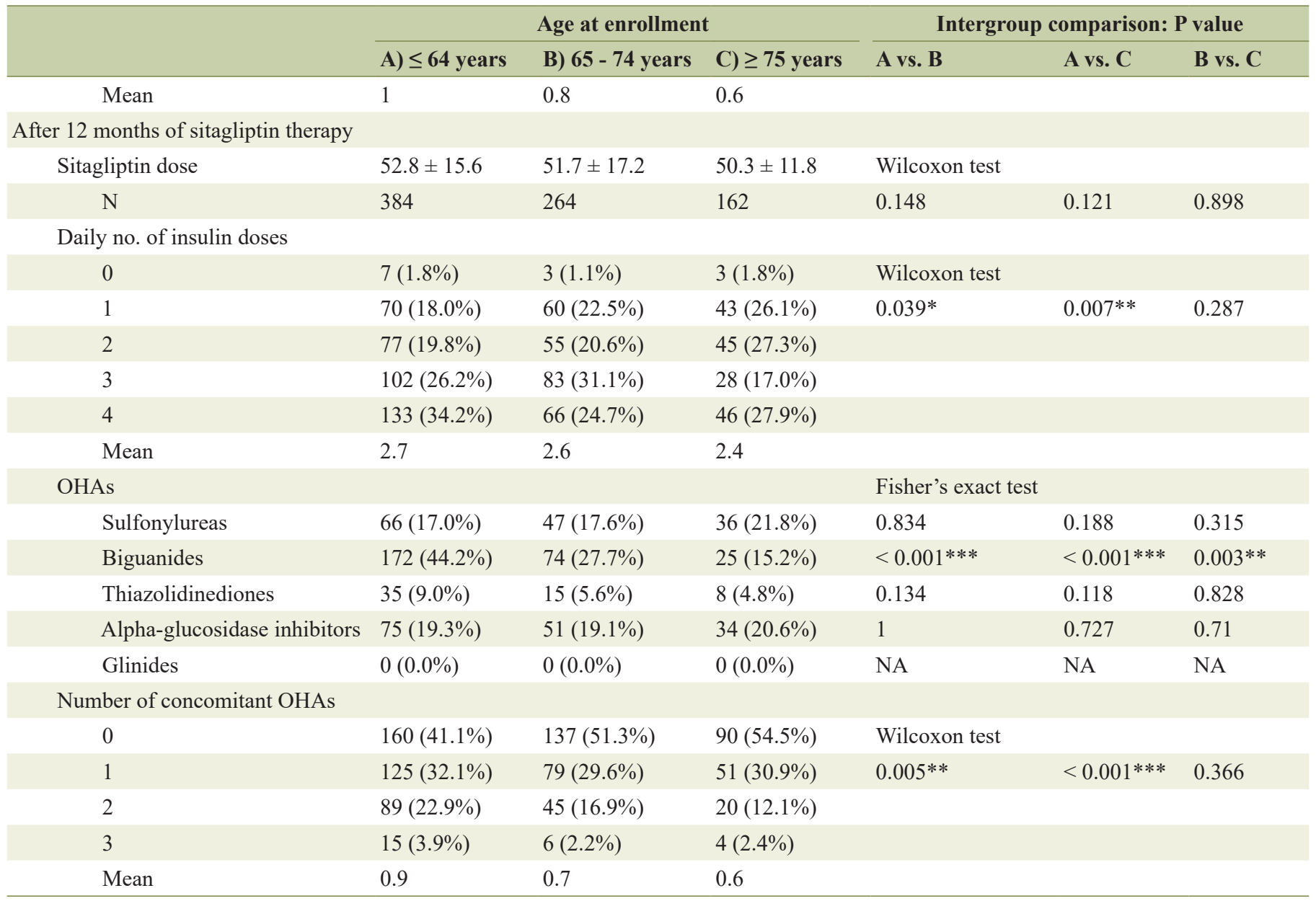

${ }^{*} \mathrm{P}<0.050,{ }^{* *} \mathrm{P}<0.010$, and ${ }^{* * *} \mathrm{P}<0.001$. Data are shown as the mean \pm standard deviation unless otherwise noted. NA: not applicable; OHA: oral hypoglycemic agent.

$8.24 \%$ to $7.64 \%$ in the elderly group, and from $8.19 \%$ to $7.66 \%$ in the very elderly group. The mean change in HbA1c at 12 months (mean \pm standard deviation (SD)) was $-0.62 \pm 1.32 \%$, $-0.60 \pm 0.92 \%$, and $-0.52 \pm 1.31 \%$, respectively. The HbA1c values at 3, 6 and 12 months after initiation of sitagliptin administration showed significant reduction compared with the baseline value, while no significant difference was observed in

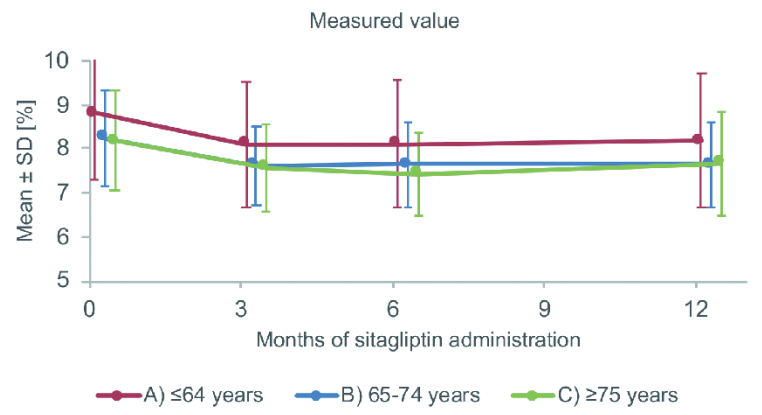

the change of HbA1c among the three groups.

\section{Changes of body weight}

Data on body weight are displayed in Figure 2. After 12 months of sitagliptin treatment, the mean body weight showed minor

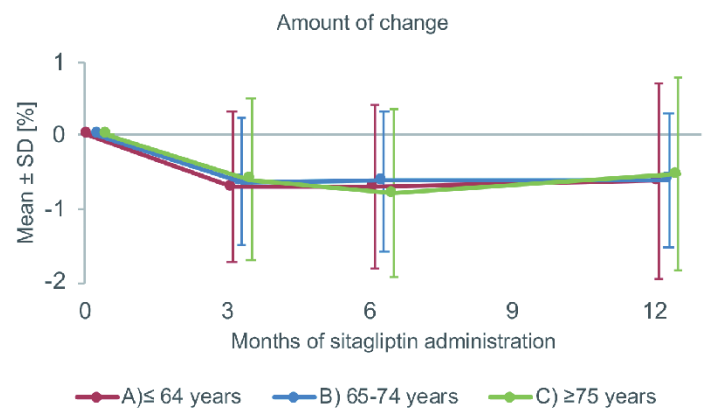

Figure 1. Changes of HbA1c. SD: standard deviation. 

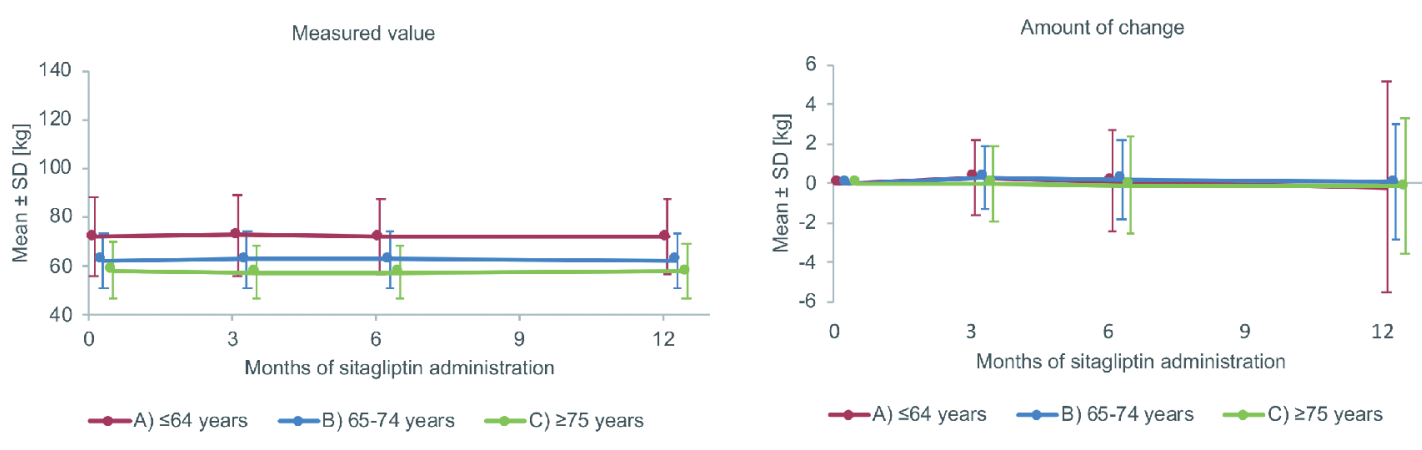

Figure 2. Changes of body weight. SD: standard deviation.
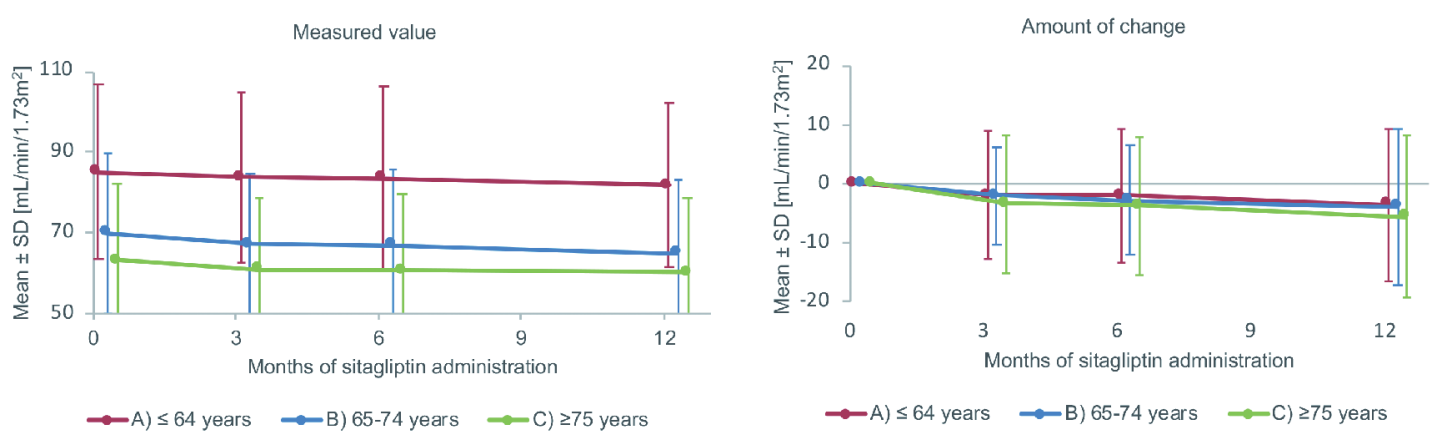

Figure 3. Changes of eGFR. SD: standard deviation.

variation from $72.1 \mathrm{~kg}$ to $71.9 \mathrm{~kg}$ in the non-elderly group, $62.4 \mathrm{~kg}$ to $62.5 \mathrm{~kg}$ in the elderly group, and $58.2 \mathrm{~kg}$ to 58.0 $\mathrm{kg}$ in the very elderly group. The mean change in body weight at 12 months (mean \pm SD) was $-0.17 \pm 5.29 \mathrm{~kg}, 0.09 \pm 2.91$ $\mathrm{kg}$, and $-0.13 \pm 3.42 \mathrm{~kg}$, respectively. There was no significant change in body weight at 12 months in any group and also no significant difference in the extent of body weight change.

\section{Changes of eGFR}

Figure 3 shows the changes of eGFR. Between baseline and 12 months, mean eGFR ( $\mathrm{mL} / \mathrm{min} / 1.73 \mathrm{~m}^{2}$ ) declined from 85.1 to 81.8 in the non-elderly group, from 70.0 to 65.1 in the elderly group, and from 63.2 to 60.3 in the very elderly group. The change of eGFR at 3, 6 and 12 months (mean \pm SD (P values by the one-sample $t$-test $)$ ) was respectively $-2.0 \pm 10.9(\mathrm{P}=$
$0.005),-2.1 \pm 11.2(\mathrm{P}=0.004)$, and $-3.6 \pm 12.9(\mathrm{P}<0.001)$ in the non-elderly group. In addition, the change of eGFR at these times was respectively $-2.1 \pm 8.3(\mathrm{P}=0.003),-2.8 \pm 9.2$ $(\mathrm{P}<0.001)$, and $-4.0 \pm 13.2(\mathrm{P}=0.002)$ in the elderly group, while it was respectively $-3.4 \pm 11.7(\mathrm{P}=0.003),-3.8 \pm 11.6(\mathrm{P}$ $<0.001)$, and $-5.7 \pm 13.7(\mathrm{P}<0.001)$ in the very elderly group. eGFR showed a significant decrease at 12 months compared with baseline in all three groups, and there were no significant intergroup differences in the changes of eGFR.

\section{Adverse events}

Among the 821 patients analyzed, severe hypoglycemia, gastrointestinal symptoms, constipation, and other events occurred in 24 patients $(2.9 \%)$, nine patients $(1.1 \%), 20$ patients $(2.4 \%)$, and 20 patients $(2.4 \%)$, respectively (Table 4$)$. There

Table 4. Adverse Events

\begin{tabular}{|c|c|c|c|c|c|c|c|}
\hline & \multirow{2}{*}{ All patients } & \multicolumn{3}{|c|}{ Age at enrollment } & \multicolumn{3}{|c|}{ Intergroup comparison: P value } \\
\hline & & A) $\leq 64$ years & B) 65 - 74 years & C) $\geq 75$ years & A vs. B & A vs. C & B vs. C \\
\hline No. of patients analyzed & $821(100.0 \%)$ & $389(100.0 \%)$ & $267(100.0 \%)$ & $165(100.0 \%)$ & \multicolumn{3}{|c|}{ Fisher's exact test } \\
\hline Gastrointestinal symptoms & $9(1.1 \%)$ & $5(1.3 \%)$ & $3(1.1 \%)$ & $1(0.6 \%)$ & 1.000 & 0.675 & 1.000 \\
\hline Constipation & $20(2.4 \%)$ & $9(2.3 \%)$ & $4(1.5 \%)$ & $7(4.2 \%)$ & 0.575 & 0.266 & 0.114 \\
\hline
\end{tabular}


were no significant intergroup differences in the incidence of any adverse events.

\section{Discussion}

The efficacy of sitagliptin as add-on therapy in T2DM patients receiving insulin has already been verified, but little has been reported about its use in elderly patients. Therefore, we compared the efficacy and safety of sitagliptin between elderly and non-elderly T2DM patients receiving insulin in routine medical practice.

In a previous study of sitagliptin for T2DM patients managed by diabetologists (ASSIST-K), 937 patients received addon sitagliptin therapy. In the present study, we analyzed 821 of these patients with $\mathrm{HbAlc}$ data up to 12 months. Demographic factors (complications and duration of diabetes) were similar between the original 937 patients and the 821 patients we analyzed, suggesting that it was reasonable to extract these patients for assessment of sitagliptin as add-on therapy to insulin.

Age-stratified comparison of demographic factors at baseline showed that elderly patients (aged $\geq 65$ years) had more complications, a longer duration of diabetes, and a lower body weight, HbA1c, and eGFR compared with non-elderly patients. Thus, there were intergroup differences in demographic factors, as would be expected.

With regard to treatment of diabetes, the elderly patients received a lower dose of sitagliptin, fewer daily insulin injections, and fewer concomitant OHAs at all times of assessment. There were significant differences between the concomitant OHAs used by elderly and non-elderly patients. Accordingly, significant differences were noted between the elderly and non-elderly patients with respect to both their demographic factors and treatment of diabetes.

After 12 months of sitagliptin treatment, HbA1c was significantly reduced from the baseline level in all three groups, with no significant intergroup differences in the extent of its reduction. We found that eGFR also decreased significantly from baseline to 12 months in all groups, again with no significant intergroup differences in the change. In contrast, there was no significant change in body weight in any group. With regard to adverse events, there were no significant intergroup differences in the incidence of severe hypoglycemia, gastrointestinal symptoms, and constipation. These efficacy and safety data for sitagliptin corresponded with those obtained by a previous study performed in insulin-naive T2DM patients (ASSET-K) [14].

The present study had some limitations. First, it was a retrospective observational study without a control group. Second, information on concomitant drugs other than hypoglycemic agents was not obtained.

In conclusion, efficacy and safety were found to be acceptable in all three age groups of T2DM patients receiving sitagliptin as add-on therapy to insulin during routine management, despite intergroup differences in demographic factors and antidiabetic agents. Accordingly, add-on therapy with sitagliptin showed similar efficacy and safety in both elderly T2DM patients and younger patients.

\section{Acknowledgments}

The authors would like to thank the members of the Kanagawa Physicians Association. The authors also thank Shogo Ito, Yoshio Aoyagi, Yasuyuki Jin, Taro Asakura, Kazuaki Shinoda, and Hideki Aizawa for their efforts in enrolling patients in the study. This research, entitled "A Study of Safety and Efficacy of Sitagliptin Added to Insulin Therapy in the Treatment of Type 2 Diabetes in Kanagawa (ASSIST-K)" was conducted by the Diabetes Task Force of the Kanagawa Physicians Association and was supported by the Kidney Foundation, Japan.

\section{Financial Disclosure or Funding}

This study was financially supported by funds from the Kidney Foundation, Japan and a grant from the Japan Renal Foundation.

\section{Conflict of Interest}

The authors declare no conflicts of interest.

\section{References}

1. Scheen AJ. A review of gliptins for 2014. Expert Opin Pharmacother. 2015;16(1):43-62.

2. Kaku K. First novel once-weekly DPP-4 inhibitor, trelagliptin, for the treatment of type 2 diabetes mellitus. Expert Opin Pharmacother. 2015;16(16):2539-2547.

3. Evans PM, Bain SC. Omarigliptin for the treatment of type 2 diabetes mellitus. Expert Opin Pharmacother. 2016;17(14):1947-1952.

4. Aroda VR, Henry RR, Han J, Huang W, DeYoung MB, Darsow T, Hoogwerf BJ. Efficacy of GLP-1 receptor agonists and DPP-4 inhibitors: meta-analysis and systematic review. Clin Ther. 2012;34(6):1247-1258 e1222.

5. Craddy P, Palin HJ, Johnson KI. Comparative effectiveness of dipeptidylpeptidase- 4 inhibitors in type 2 diabetes: a systematic review and mixed treatment comparison. Diabetes Ther. 2014;5(1):1-41.

6. Scheen AJ. Safety of dipeptidyl peptidase-4 inhibitors for treating type 2 diabetes. Expert Opin Drug Saf. 2015;14(4):505-524.

7. Plosker GL. Sitagliptin: a review of its use in patients with type 2 diabetes mellitus. Drugs. 2014;74(2):223242.

8. Lee M, Rhee MK. Sitagliptin for Type 2 diabetes: a 2015 update. Expert Rev Cardiovasc Ther. 2015;13(6):597610 .

9. Maeda H, Kubota A, Tanaka Y, Terauchi Y, Matsuba I, Asset- K. Study group. The safety, efficacy and predictors for $\mathrm{HbAlc}$ reduction of sitagliptin in the treatment of Japanese type 2 diabetes. Diabetes Res Clin Pract. 2012;95(1):e20-22. 
10. Kubota A, Maeda H, Kanamori A, Matoba K, Jin Y, Minagawa $\mathrm{F}$, Obana $\mathrm{M}$, et al. Pleiotropic effects of sitagliptin in the treatment of type 2 diabetes mellitus patients. J Clin Med Res. 2012;4(5):309-313.

11. Kubota A, Maeda H, Kanamori A, Matoba K, Jin Y, Minagawa $\mathrm{F}$, Obana $\mathrm{M}$, et al. Efficacy and safety of sitagliptin monotherapy and combination therapy in Japanese type 2 diabetes patients. J Diabetes Investig. 2012;3(6):503-509.

12. Kanamori A, Matsuba I. Factors associated with reduced efficacy of sitagliptin therapy: analysis of 93 patients with type 2 diabetes treated for 1.5 years or longer. J Clin Med Res. 2013;5(3):217-221.

13. Maeda H, Kubota A, Kanamori A, Tanaka Y, Terauchi Y, Matsuba I, Asset- K. Study Group. Long-term efficacy and safety of sitagliptin in the treatment of Japanese Type 2 diabetes (ASSET-K1) to a target of HbA1c $<7 \%$. J Endocrinol Invest. 2013;36(8):568-573.

14. Umezawa S, Kubota A, Maeda H, Kanamori A, Matoba $\mathrm{K}$, Jin Y, Minagawa F, et al. Two-year assessment of the efficacy and safety of sitagliptin in elderly patients with type 2 diabetes: Post hoc analysis of the ASSET-K study. BMC Endocr Disord. 2015;15:34.

15. Takai M, Ishikawa M, Maeda H, Kanamori A, Kubota A, Amemiya H, Iizuka T, et al. Safety and efficacy of adding sitagliptin to insulin in patients with type 2 diabetes: the ASSIST-K study. Diabetes Res Clin Pract. 2014;103(3):e30-33.

16. Maeda H, Kubota A, Kanamori A, Tanaka Y, Terauchi Y, Matsuba I, The Study Group of the Diabetes Committee, Kanagawa Physicians Association. Effects of sitagliptin on the serum creatinine in Japanese type 2 diabetes. Diabetes Res Clin Pract. 2015;108(3):e42-45.

17. Yuasa S, Sato K, Takai M, Ishikawa M, Umezawa S, Kubota A, Maeda H, et al. Factor analysis of changes in hemoglobin A1c after 12 months of sitagliptin therapy in patients with type 2 diabetes. J Clin Med Res. 2016;8(6):461-471.

18. Janghorbani M, Van Dam RM, Willett WC, Hu FB. Systematic review of type 1 and type 2 diabetes mellitus and risk of fracture. Am J Epidemiol. 2007;166(5):495-505.

19. Ohara T, Doi Y, Ninomiya T, Hirakawa Y, Hata J, Iwaki $\mathrm{T}$, Kanba S, et al. Glucose tolerance status and risk of dementia in the community: the Hisayama study. Neurology. 2011;77(12):1126-1134.

20. Sinclair AJ, Robert IE, Croxson SC. Mortality in older people with diabetes mellitus. Diabet Med. 1997;14(8):639647.

21. Meneilly GS. Diabetes in the elderly. Med Clin North Am. 2006;90(5):909-923.

22. Chelliah A, Burge MR. Hypoglycaemia in elderly patients with diabetes mellitus: causes and strategies for prevention. Drugs Aging. 2004;21(8):511-530. 